\title{
Simultaneous fault and input time delay estimation for an actuator system: theory and flight data validation
}

\author{
Lejun Chen, Christopher Edwards, Halim Alwi, and Masayuki Sato
}

\begin{abstract}
This paper proposes a first order sliding mode observer for the purpose of simultaneously estimating the unknown input time delay and reconstructing the loss of effectiveness in a model of an actuator. The adaptive algorithm is driven by the 'equivalent output error injection' signal associated with the sliding motion. Sufficient conditions are given to ensure finite time convergence of the state estimation error system, ensuring both the time delay estimation error and the estimation error associated with the actuator fault converge to a small region around zero. The efficacy of the approach has been evaluated via both a numerical simulation and flight data validation.
\end{abstract}

Keyword: Fault detection; Delay systems; Variablestructure/sliding-mode control

\section{INTRODUCTION}

$\mathbf{S}$ LIDING mode observers (SMOs) have been widely applied to solve Fault Detection and Diagnosis (FDD) problems [1] by exploiting the concept of the equivalent injection signals [2], [3]. In [2], an SMO was first employed for the purpose of fault reconstruction. Fault estimation as opposed to merely detection is very useful in terms of the fault tolerant control. In recent years, several authors have extended typical adaptive observer structures and included sliding mode injection terms to improve parameter estimation robustness with respect to mismatched uncertainty [5]-[7]. In particular, the work in [5] and [29] employ an adaptive law within an SMO framework for solving FDD problems.

In practical systems, time delays are inevitable due to finite signal transmission speed and the processing time associated with different system components. In many situations, to build a realistic dynamic model and achieve ideal closedloop stability performance, time delays should be explicitly considered. Many control and observation methods assume that time delays are known. However in many practical situations, it is often the case that the delays are unknown or uncertain. Time delay estimation (TDE) or time delay identification has not been widely developed in the literature due to its computational complexity. However, a number of authors have studied TDE in the last decade (e.g. see [10][23]). Using TDE, many time delay systems can be stabilised more effectively, especially in the situation where there exists

Lejun Chen is with School of Aerospace, Transport and Manufacturing, Cranfield University, UK (e-mail: Lejun.Chen@cranfield.ac.uk).

Christopher Edwards and Halim Alwi are with College of Engineering, Mathematics \& Physical Sciences, University of Exeter, UK (e-mail: C.Edwards@exeter.ac.uk,h.alwi@exeter.ac.uk).

Masayuki Sato is with Japan Aerospace Exploration Agency, Mitaka, Tokyo 181-0015, Japan (e-mail: sato.masayuki@jaxa.jp). large unknown time delays. The work in [9]-[13] exploited optimisation based ideas for TDE. However these methods are not robust enough against variations of the time delay, and some of these works assumed the unknown time delay to be monotonic. The methods proposed in [17], [18] have been applied to estimate unknown time delays, however, these methods modelled the delayed input as transport partial differential equation (PDE) boundary conditions, and constant delays were estimated via adaptive backstepping control. The theory of non-commutative rings has also been applied to the analysis of time delay systems and non-commutative ring based TDE has been proposed in [19]. In [14], a convolution-based algebraic approach was used for the estimation of constant time delays. Observer based methods have also appeared in the literature [21], [22]. The work in [21] decomposed the system states via a Taylor polynomial and created an augmented observer to estimate the constant state delay. In [22], [23], Kalmanlike observers were created to estimate the time delay for an extended system with the delay as part of the extended state. In [24] an adaptive observer was created based on Taylor's theorem. Sliding mode based methods have also been applied to TDE [25]. For instance, the recent work in [20] exploited a super twisting algorithm for the purpose of TDE. However, this work requires the input signals (affected by the time delay) to be strictly monotonic and assumes knowledge of the delayed inputs to be available - which is not realistic in practice. In [15], the time delay constant $\tau$ is modelled in the frequency domain as an exponential function $e^{\tau s}$ and a simple filter based adaptation scheme was used to identify the time delay in finite time. This approach was further extended and applied to vehicle applications [16].

The main contribution of this paper is to develop a simple adaptive sliding mode observer to simultaneously estimate an unknown input delay whilst estimating the magnitude of a multiplicative fault (i.e. loss of the actuator effectiveness) despite external disturbances. Since the unknown input delay and the multiplicative actuator faults appear in the same channel, this increases the complexity of the problem. To the authors' best knowledge, simultaneous FDD and TDE has not been addressed in the literature before. The adaptation algorithm is created via exploiting knowledge of the 'equivalent output error injection' signal. Assuming a Persistently Exciting (PE) regressor, a sufficient condition is proposed to guarantee convergence of the estimation errors. Unlike [11], [12], [20], this method does not require knowledge of the delayed input $u(t-h)$ which is not always available in practice. Numerical 
simulation results show the efficacy of the method. To further demonstrate its practicality, this approach is applied to real flight test data, collected from an actuator fault tolerant flight test campaign, to estimate both the values of the aileron input delay and the loss of actuator effectiveness.

The notation in this paper is standard. For example, $A>0$ denotes a positive definite matrix, and the symbol $\|\cdot\|$ denotes the Euclidean norm or its induced norm.

\section{OBSERVER FORMULATION}

Consider a single input, signal output actuator subject to an unknown time-variant time delay and faults modelled as

$$
\begin{aligned}
& \dot{x}(t)=a x(t)+b w(t) u(t-h(t))+d(t) \\
& y(t)=c x(t)
\end{aligned}
$$

In (1), the parameter set $(a, b, c)$ is assumed to be known. It is assumed that the actuator dynamics are stable. In (1), $h(t)$ represents an unknown time delay which satisfies $h(t) \in$ $\left[\begin{array}{ll}h_{\min } & h_{\max }\end{array}\right]$, and $x(\tau)=x(0)$ for all $\tau \in[-h, 0]$. The quantities $x(t) \in \mathbb{R}$ and $u(t) \in \mathbb{R}$ in (1) denote the actuator internal state and the command input respectively. Here it is assumed that the state $x(t) \in \mathbb{R}$ is measurable. In (1), $d(t)$ captures the disturbance which satisfies $|d(t)| \leq \bar{d}$ where $\bar{d}$ is a known scalar. The unknown scalar function $w(t) \in\left[\begin{array}{ll}0 & 1\end{array}\right]$ represents the effectiveness level of the actuator which is unknown and to be estimated [1]. If $0<w(t)<1$, the actuator behaves with reduced effectiveness (i.e. some level of fault is present). For a fault-free actuator $w=1$, and for a completely failed actuator $w(t)=0$. In (1), both $h(t)$ and $w(t)$ are to be estimated.

Assumption 2.1: It is assumed that

i) $a<0$ (i.e. the actuator dynamics are stable);

ii) the scalar functions $w(t)$ and $h(t)$ are slowly varying, i.e. $\dot{w}(t) \approx 0, \dot{h}(t) \approx 0$

iii) the input $u(t)$ is continuous and bounded, and satisfies $|u(t)| \leq \bar{u}$ for all $t \in\left[-h_{\max } \infty\right)$;

iv) $u(t)$ has a derivative with Lipschitz's constant $C$ for all $t \in\left[\begin{array}{ll}-h_{\max } & \infty\end{array}\right)$

Remark 2.1: Assumption 2.1 ii) has been widely used for the development of adaptive schemes in the literature [8]. This assumption is usually made to ensure the exponentially stability of the parameter estimation error to zero.

The proposed sliding mode observer for the system in (1) has the following structure

$$
\dot{\hat{x}}(t)=a \hat{x}(t)+b \hat{w}(t) \hat{h}(t) \dot{u}(t-\hat{h})+g_{l} e_{y}(t)+\nu(t)
$$

where $\hat{x}(t)$ represents the estimate of $x(t)$. In (2) the output estimation error is $e_{y}(t):=c(x(t)-\hat{x}(t))$ and the nonlinear output error injection vector

$$
\nu(t)=\left\{\begin{array}{cl}
k(t) \operatorname{sign}\left(e_{y}\right) & \text { if } e_{y} \neq 0 \\
0 & \text { otherwise }
\end{array}\right.
$$

where the modulation gain $k(t)$ is a positive scalar function which will be defined later and $g_{l}$ denotes the observer gain to be selected. In (2) $\hat{w}(t)$ and $\hat{h}(t)$ are estimates to be constructed via the adaptation scheme defined in the sequel.
The term $\dot{u}(t-\hat{h})$ in (2) represents the first derivative of $u(t-\hat{h})$. Here the aim is to create $\hat{w}(t)$ and $\hat{h}(t)$ so that $e_{y}(t) \rightarrow 0$ in finite time.

Remark 2.2: Assumption 2.1 (iii) implies that the input $u(t-$ $\hat{h})$ is also bounded. In $(2) \dot{u}(t-\hat{h})$ can be estimated using a robust exact differentiator [26] defined as

$$
\begin{aligned}
\dot{x}_{s t} & =\nu_{s t} \\
\nu_{s t} & =\nu_{s t, 1}-k_{1}\left|x_{s t}-u(t-\hat{h})\right|^{\frac{1}{2}} \operatorname{sign}\left(x_{s t}-u(t-\hat{h})\right) \\
\dot{\nu}_{s t, 1} & =-k_{2} \operatorname{sign}\left(x_{s t}-u(t-\hat{h})\right)
\end{aligned}
$$

where $k_{1}$ and $k_{2}$ are positive scalars. In [26] the following sufficient conditions for the convergence of the 'super-twisting' algorithm are given as

$$
k_{2}>C \text { and } k_{1}^{2} \geq 4 C \frac{k_{2}+C}{k_{2}-C}
$$

where the Lipschitz constant $C$ defined in Assumption 2.1 (iv) is exploited.

\section{REACHING AND SLIDING MOTION ANALYSIS}

In this section, the state estimation error will be analysed to demonstrate a sliding motion takes place in finite time.

Define the state estimation error $e(t)=x(t)-\hat{x}(t)$, then from (1) and (2) it follows

$\dot{e}(t)=\tilde{a} e(t)+b w(t) u(t-h)-b \hat{w}(t) \hat{h}(t) \dot{u}(t-\hat{h})-\nu(t)+d(t)$

where $\tilde{a}=a-g_{l} c$.

Proposition 1: If the modulation gain $k(t)$ satisfies

$$
k(t) \geq b \bar{u}+b|\hat{w}(t)||\hat{h}(t)||\dot{u}(t-\hat{h})|+\bar{d}+\eta
$$

where $\bar{u}$ represents a bound on the delayed input from Assumption 2.1 and $\eta$ is a positive scalar, then $e_{y}$ converges to zero and a sliding motion on $e_{y}=0$ will take place for all $t \geq t_{0}$ where $t_{0}$ is the finite time at which sliding is established.

Proof 1: Define $\tilde{V}\left(e_{y}\right)=\frac{1}{2} e_{y}^{2}(t)$ then it follows from the expression for $\dot{e}(t)$ in (6) that

$\dot{\tilde{V}}=e^{2} c^{2} \tilde{a}+e c^{2}(b w(t) u(t-h)-b \hat{w}(t) \hat{h}(t) \dot{u}(t-\hat{h})-\nu(t)+d(t))$

Here it is assumed without loss of generality (w.l.o.g) $c>0$. Since $(a, c)$ are scalars, there always exists $g_{l}$ such that $\tilde{a}<0$ and therefore

$$
\dot{\tilde{V}} \leq e c^{2}(b w(t) u(t-h)-b \hat{w}(t) \hat{h}(t) \dot{u}(t-\hat{h})-\nu(t)+d(t))
$$

Using the fact $|w(t)| \leq 1,|u(t-h)| \leq \bar{u}$ and $|d(t)| \leq \bar{d}$

$$
\dot{\tilde{V}} \leq|e| c^{2}(b \bar{u}+b|\hat{w}(t)||\hat{h}(t)||\dot{u}(t-\hat{h})|-k(t)+\bar{d})
$$

If (7) is satisfied, $\dot{\tilde{V}} \leq-\eta|c|\left|e_{y}\right| \leq-\eta|c| \sqrt{2 \tilde{V}}$ and therefore $e_{y}$ will converge to zero and a sliding motion will take place in finite time.

During sliding on $e_{y}=0, \dot{e}(t)=e(t)=0$. Substituting these quantities into (6) yields

$$
\nu_{e q}(t)=b(w(t) u(t-h)-\hat{w}(t) \hat{h}(t) \dot{u}(t-\hat{h}))+d(t)
$$

where the quantity $\nu_{e q}(t)$ is the equivalent output error injection signal necessary to maintain sliding [3]. Here the signal 
$\nu_{e q}(t)$ is obtained by replacing the signum function with a sigmoid function [2] in the signal $\nu(t)$, and therefore the discontinuity is approximated using a smoothing factor. Using Taylor's theorem, the following equality is satisfied

$$
u(t-h)=u(t-\hat{h})-\tilde{h}(t) \dot{u}(t-\hat{h})+o(\tilde{h})
$$

where $\tilde{h}(t)=h-\hat{h}(t)$ denotes the time delay estimation error.

Substituting (12) into (11) yields

$$
\begin{aligned}
\nu_{e q}(t)= & b(w(t) u(t-\hat{h})-\tilde{h}(t) w(t) \dot{u}(t-\hat{h})-\hat{w}(t) \hat{h}(t) \dot{u}(t-\hat{h})) \\
& +o(\tilde{h})+d(t)
\end{aligned}
$$

Define the fault estimation error as $\tilde{w}(t)=w(t)-\hat{w}(t)$, then (13) is equivalent to

$$
\begin{aligned}
\nu_{e q}(t)= & o(\tilde{h})+d(t)+b(w(t) u(t-\hat{h})-\tilde{h}(t) \tilde{w}(t) \dot{u}(t-\hat{h}) \\
& -\tilde{h}(t) \hat{w}(t) \dot{u}(t-\hat{h})-\hat{w}(t) \hat{h}(t) \dot{u}(t-\hat{h})) \\
= & o(\tilde{h})+d(t)+b(w(t) u(t-\hat{h})-\tilde{h}(t) \tilde{w}(t) \dot{u}(t-\hat{h}) \\
& -(h-\hat{h}(t)) \hat{w}(t) \dot{u}(t-\hat{h})-\hat{w}(t) \hat{h}(t) \dot{u}(t-\hat{h})) \\
= & o(\tilde{h})+d(t)+b(w(t) u(t-\hat{h})-\tilde{h}(t) \tilde{w}(t) \dot{u}(t-\hat{h}) \\
& -h \hat{w}(t) \dot{u}(t-\hat{h}))
\end{aligned}
$$

Define a lumped uncertainty as

$$
\xi(t):=-b \tilde{h}(t) \tilde{w}(t) \dot{u}(t-\hat{h})+o(\tilde{h})+d(t)
$$

If the sliding motion on $e_{y}=0$ exists and can be maintained, the signals $\hat{h}(t)$ and $\hat{w}(t)$ are available from the adaptation scheme given later. Since $\tilde{h}=h-\hat{h}$ and $h \in\left[\begin{array}{ll}h_{\min } & h_{\max }\end{array}\right]$,

$$
|\tilde{h}| \leq|h|+|\hat{h}| \leq h_{\max }+|\hat{h}|
$$

Also since $\tilde{w}=w-\hat{w}$ and $w \in\left[\begin{array}{ll}0 & 1\end{array}\right]$,

$$
|\tilde{w}| \leq|w|+|\hat{w}| \leq 1+|\hat{w}|
$$

From a practical engineering perspective, the approximation of $o(\tilde{h})$ in $(15)$ stops at order one and $o(\tilde{h})$ is assumed to be negligible. Since the values of $h_{\max }, \bar{d}$ and $b$ are known,

$$
\|\xi(t)\| \leq \bar{\xi}(t)
$$

where $\bar{\xi}(t)$ is known and is given explicitly as

$$
\bar{\xi}(t)=b\left(h_{\max }+\hat{h}(t)\right)(1+\hat{w}(t))|\dot{u}(t-\hat{h})|+\bar{d}
$$

Note: it can be seen from (15) that if the fault estimate error $\tilde{w}(t)$ and the time delay estimate error $\tilde{h}(t)$ converge to zero, $\xi(t)$ approaches a residual set around zero bounded by $\bar{d}$. In particular, in the situation when $d(t)=0, \xi(t) \rightarrow 0$.

Now define

$$
\phi(t):=\left[\begin{array}{l}
w(t) \\
h(t)
\end{array}\right] \text { and } \psi(t):=\left[\begin{array}{c}
b u(t-\hat{h}) \\
-b \hat{w}(t) \dot{u}(t-\hat{h})
\end{array}\right]
$$

where $\phi(t)$ is the vector of unknown parameters to be estimated and $\psi(t)$ is a known regressor function. Consequently (14) can be written in the form of

$$
\nu_{e q}(t)=\psi^{T}(t) \phi(t)+\xi(t)
$$

An adaptive law will now be developed to describe the evolution of $\phi(t)$ driven by the equivalent injection $\nu_{e q}(t)$.

Assumption 3.1: It is assumed that the regressor function $\psi(t)$ remains bounded so that $\|\psi(t)\|<\psi_{0}$ (where the positive scalar $\psi_{0}$ is known) and is Persistent Exciting (PE), that is for any $t \in \mathbb{R}$

$$
\int_{t}^{t+\Delta t} \psi(s) \psi^{T}(s) d s \geq \epsilon I_{2}
$$

where $\Delta t>0$ and $\epsilon>0$.

Although the scalar $b$ in $\psi(t)$ is given, it can scaled to any non-zero constant to adjust PE without changing the actuator dynamic, which can be achieved w.l.o.g by a state transformation of (1) if necessary.

Remark 3.1: As argued in [8], if $\psi(t)$ is PE, the following adaptation law $\dot{\hat{\phi}}(t)=\gamma \psi(t)\left(\nu_{e q}(t)-\psi^{T}(t) \hat{\phi}\right)$ ensures the estimation error $\tilde{\phi}(t)=\phi(t)-\hat{\phi}(t)$ is globally exponentially stable at the origin provided that $\xi(t) \equiv 0$. In the situation when $\xi(t)$ is essentially bounded, the estimation error is input-to-state stable. However, this adaptation law does not guarantee a finite time stability.

\section{CONVERGENCE ANALYSIS}

In this paper, an alternative adaptation law based on ideas from [15] is used to ensure the finite time convergence of the estimation errors. Furthermore, the modulation function and the bound of estimation error $\tilde{\phi}(t)$ will be calculated explicitly in the sequel.

Define a non-homogeneous matrix differential equations as

$$
\dot{X}(t)=-\lambda_{0} X(t)+\psi(t) \psi^{T}(t) \quad X(0)=0
$$

where $X(t) \in \mathbb{R}^{2 \times 2}$ where $\lambda_{0}$ is a positive scalar. Explicitly the solutions of (23) is

$$
X(t)=\int_{0}^{t} e^{-\lambda_{0}(t-\tau)} \psi(\tau) \psi^{T}(\tau) d \tau
$$

Remark 4.1: Since the regressor function $\psi(t)$ is PE, the solution $X(t)$ in (24) implies that $X(t)$ and $X^{-1}(t)$ are positive definite for any $t \in \mathbb{R}$. Here it is assumed, w.l.o.g, $\lambda_{\min }(X(t))>\beta$ where $\beta$ is a positive scalar [15].

Also define a vector $\chi(t) \in \mathbb{R}^{2}$ as the solution of

$$
\dot{\chi}(t)=-\lambda_{0} \chi(t)+\psi(t) \nu_{e q}(t) \quad \chi(0)=0
$$

so that

$$
\chi(t)=\int_{0}^{t} e^{-\lambda_{0}(t-\tau)} \psi(\tau) \nu_{e q}(\tau) d \tau
$$

Finally define

$$
\dot{\zeta}(t)=-\lambda_{0} \zeta(t)+\psi(t) \xi(t) \quad \zeta(0)=0
$$

so that

$$
\zeta(t):=\int_{0}^{t} e^{-\lambda_{0}(t-\tau)} \psi(\tau) \xi(\tau) d \tau
$$

Define a new scalar variable $\bar{\zeta}(t)$ as the solution of

$$
\dot{\bar{\zeta}}(t)=-\lambda_{0} \bar{\zeta}(t)+\psi_{0} \bar{\xi}(t) \quad \bar{\zeta}(0)=0
$$

where $\bar{\xi}(t)$ is defined in (19). Then it follows

$$
\|\zeta(t)\|<\bar{\zeta}(t)
$$

where $\bar{\zeta}(t)$ is known since $\psi_{0}$ and $\bar{\xi}(t)$ is known. Using the expression in (21), it follows from (24), (26) and (28) that

$$
\chi(t)=X(t) \phi(t)+\zeta(t)
$$


Define a switching function $s(t) \in \mathbb{R}^{2}$ as

$$
s(t):=\hat{\phi}(t)-X^{-1}(t) \chi(t)
$$

where $\hat{\phi}(t)$ represents the estimate of $\phi(t)$ to be constructed from the adaptation law which will be defined in the sequel. Substituting (31) into (32) yields

$$
s(t)=\hat{\phi}(t)-\phi(t)-X^{-1}(t) \zeta(t)=-\tilde{\phi}(t)-X^{-1}(t) \zeta(t)
$$

where $\tilde{\phi}(t)$ is the parameter estimation error.

Proposition 2: Choosing the adaptation scheme as

$$
\dot{\hat{\phi}}(t)=-\rho(t) \frac{s(t)}{\|s(t)\|}
$$

where the (known) modulation function $\rho(t)$ takes the form

$$
\rho(t)=\frac{\psi_{0}^{2}}{\beta^{2}}\left(2-e^{-\lambda_{0} t}\right)+\frac{1}{\beta}\left(\lambda_{0} \bar{\zeta}(t)+\psi_{0} \bar{\xi}(t)\right)+\eta_{0}
$$

where $\bar{\zeta}(t)$ is the solution of (29). Then a sliding motion is forced to exist on $s \equiv 0$ in finite time $t_{s}$.

Proof 2: Define a Lyapunov candidate as $V=\frac{1}{2} s^{T}(t) s(t)$. Exploiting Assumption 2.1 ii), the derivative of $V$ is

$$
\begin{aligned}
\dot{V} & =s^{T}(t) \dot{s}(t)=s^{T}(t) \frac{d}{d t}\left(-\tilde{\phi}(t)-X^{-1}(t) \zeta(t)\right) \\
& =s^{T}(t)\left(-\dot{\tilde{\phi}}(t)+X^{-1}(t) \dot{X}(t) X^{-1}(t) \zeta(t)-X^{-1}(t) \dot{\zeta}(t)\right) \\
& =s^{T}(t)\left(\dot{\hat{\phi}}(t)+X^{-1}(t) \dot{X}(t) X^{-1}(t) \zeta(t)-X^{-1}(t) \dot{\zeta}(t)\right)
\end{aligned}
$$

From (23), the term $X^{-1}(t) \dot{X}(t) X^{-1}(t)$ in (36) satisfies

$$
\left\|X^{-1}(t) \dot{X}(t) X^{-1}(t)\right\| \leq \frac{\psi_{0}^{2}}{\beta^{2}}\left(2-e^{-\lambda_{0} t}\right)
$$

and from (30) the term $X^{-1}(t) \dot{\zeta}(t)$ is bounded by

$$
\left\|X^{-1}(t) \dot{\zeta}(t)\right\| \leq\left\|X^{-1}(t)\right\|\|\dot{\zeta}(t)\| \leq \frac{1}{\beta}\left(\lambda_{0} \bar{\zeta}(t)+\psi_{0} \bar{\xi}(t)\right)
$$

Substituting (34) into (36) and using (37) and (38) yields

$$
\begin{aligned}
\dot{V} & =-\rho(t)\|s(t)\|+s^{T}(t)\left(X^{-1}(t) \dot{X}(t) X^{-1}(t) \zeta(t)-X^{-1}(t) \dot{\zeta}(t)\right) \\
& \leq\left(-\rho(t)+\frac{\psi_{0}^{2}}{\beta^{2}}\left(2-e^{-\lambda_{0} t}\right)+\frac{1}{\beta}\left(\lambda_{0} \bar{\zeta}(t)+\psi_{0} \bar{\xi}(t)\right)\right)\|s(t)\|
\end{aligned}
$$

Therefore by choosing $\rho(t)$ as in (35) yields

$$
\dot{V} \leq-\eta_{0}\|s(t)\|=-\eta_{0} \sqrt{2 V}
$$

and therefore $s(t) \rightarrow 0$ in finite time and

$$
\tilde{\phi}(t)=-X^{-1}(t) \zeta(t)
$$

holds in finite time $t_{s}$.

Remark 4.2: The global bound on $\xi(t)$ given by $\bar{\xi}(t)$ in (19) is very conservative. In a neighbourhood of $\tilde{\phi}(t)=0$, a less conservative bound

$$
\|\xi(t)\|<d_{0}\|\tilde{\phi}(t)\|+d_{1}
$$

can be used (where $d_{0}$ and $d_{1}$ are fixed positive scalars). From the definition of $\xi(t)$ in (15), inequality (42) better reflects the inherent property that as $\tilde{\phi}(t) \rightarrow 0$, the lumped uncertainty $\xi(t) \rightarrow d(t)$ as observed in (15).

The following proposition analyzes the evolution of the parameter estimation error during the sliding motion in $s=0$.

Proposition 3: If $\beta \lambda_{0}>\psi_{0} d_{0}$, then during sliding when (41) is satisfied, $\tilde{\phi}(t)$ is bounded for all $t \geq t_{s}$.

Proof 3: From (28) and (42)

$$
\begin{aligned}
\|\zeta(t)\| & \leq \int_{t_{s}}^{t} e^{-\lambda_{0}(t-\tau)}\|\psi(\tau)\|\|\xi(\tau)\| d \tau \\
& \leq \int_{t_{s}}^{t} e^{-\lambda_{0}(t-\tau)} \psi_{0} d_{0}\|\tilde{\phi}(\tau)\| d \tau+\int_{t_{s}}^{t} e^{-\lambda_{0}(t-\tau)} \psi_{0} d_{1} d \tau
\end{aligned}
$$

During sliding $s(t)=0$ and from (41), $\tilde{\phi}(t)=-X^{-1}(t) \zeta(t)$. Therefore $\|\tilde{\phi}(t)\| \leq\left\|X^{-1}(t)\right\|\|\zeta(t)\|<\frac{1}{\beta}\|\zeta(t)\|$ since $\|X(t)\|>\beta$. Let $\mu:=\frac{\psi_{0} d_{0}}{\beta}$ and therefore using (43)

$$
\|\tilde{\phi}(t)\| \leq\left\|\tilde{\phi}\left(t_{s}\right)\right\|+\int_{t_{s}}^{t} e^{-\lambda_{0}(t-\tau)}\left(\mu\|\tilde{\phi}(\tau)\|+\frac{\psi_{0} d_{1}}{\beta}\right) d \tau
$$

Define $\bar{\omega}(t):=e^{\lambda_{0} t}\|\tilde{\phi}(t)\|$, then from (44)

$$
\begin{aligned}
\bar{\omega}(t) & \leq e^{\lambda_{0} t}\left\|\tilde{\phi}\left(t_{s}\right)\right\|+\mu \int_{t_{s}}^{t} \bar{\omega}(\tau) d \tau+\int_{t_{s}}^{t} e^{\lambda_{0} \tau} \frac{\psi_{0} d_{1}}{\beta} d \tau \\
& =\mu \int_{t_{s}}^{t} \bar{\omega}(\tau) d \tau+\underbrace{\frac{\psi_{0} d_{1}}{\lambda_{0} \beta}\left(e^{\lambda_{0} t}-e^{\lambda_{0} t_{s}}\right)+e^{\lambda_{0} t}\left\|\tilde{\phi}\left(t_{s}\right)\right\|}_{\lambda(t)}
\end{aligned}
$$

which is in the integral form of the Bellman-Gronwall Lemma. Using the Bellman-Gronwall Lemma [28]

$$
\bar{\omega}(t) \leq \lambda(t)+\mu \int_{t_{s}}^{t} \lambda(\tau) e^{\mu(t-\tau)} d \tau
$$

The integral term in (46) can be written as

$$
\begin{aligned}
\int_{t_{s}}^{t} \lambda(\tau) e^{\mu(t-\tau)} d \tau= & \int_{t_{s}}^{t} \frac{\psi_{0} d_{1}}{\lambda_{0} \beta}\left(e^{\lambda_{0} \tau}-e^{\lambda_{0} t_{s}}\right) e^{\mu(t-\tau)} d \tau \\
& +\int_{t_{s}}^{t}\left\|\tilde{\phi}\left(t_{s}\right)\right\| e^{\lambda_{0} \tau} e^{\mu(t-\tau)} d \tau \\
= & \frac{\psi_{0} d_{1}}{\lambda_{0} \beta} e^{\mu t}\left(\int_{t_{s}}^{t} e^{\left(\lambda_{0}-\mu\right) \tau} d \tau-\int_{t_{s}}^{t} \frac{e^{\lambda_{0} t_{s}}}{e^{\mu \tau}} d \tau\right) \\
& +\left\|\tilde{\phi}\left(t_{s}\right)\right\| e^{\mu t} \int_{t_{s}}^{t} e^{\left(\lambda_{0}-\mu\right) \tau} d \tau \\
= & \frac{\psi_{0} d_{1}}{\lambda_{0} \beta} e^{\mu t}\left(\frac{e^{\left(\lambda_{0}-\mu\right) t}-e^{\left(\lambda_{0}-\mu\right) t_{s}}}{\lambda_{0}-\mu}+\frac{e^{\lambda_{0} t_{s}}}{\mu e^{\mu t}}\right. \\
& \left.-\frac{e^{\lambda_{0} t_{s}}}{\mu e^{\mu t_{s}}}\right)+\left\|\tilde{\phi}\left(t_{s}\right)\right\| e^{\mu t} \frac{e^{\left(\lambda_{0}-\mu\right) t}-e^{\left(\lambda_{0}-\mu\right) t_{s}}}{\lambda_{0}-\mu}
\end{aligned}
$$

Substituting (47) into (46) yields

$$
\begin{aligned}
e^{\lambda_{0} t}\|\tilde{\phi}(t)\| \leq & \frac{\mu \psi_{0} d_{1}}{\lambda_{0} \beta} e^{\mu t}\left(\frac{e^{\left(\lambda_{0}-\mu\right) t}-e^{\left(\lambda_{0}-\mu\right) t_{s}}}{\lambda_{0}-\mu}+\frac{e^{\lambda_{0} t_{s}}}{\mu e^{\mu t}}-\frac{e^{\lambda_{0} t_{s}}}{\mu e^{\mu t_{s}}}\right) \\
& +\frac{\psi_{0} d_{1}}{\lambda_{0} \beta}\left(e^{\lambda_{0} t}-e^{\lambda_{0} t_{s}}\right)+e^{\lambda_{0} t}\left\|\tilde{\phi}\left(t_{s}\right)\right\| \\
& +\mu\left\|\tilde{\phi}\left(t_{s}\right)\right\| e^{\mu t} \frac{e^{\left(\lambda_{0}-\mu\right) t}-e^{\left(\lambda_{0}-\mu\right) t_{s}}}{\lambda_{0}-\mu}
\end{aligned}
$$


Multiplying $e^{-\lambda_{0} t}$ on both sides of (48) yields

$$
\begin{aligned}
\|\tilde{\phi}(t)\| \leq & \frac{\mu \psi_{0} d_{1}}{\lambda_{0} \beta} e^{\left(\mu-\lambda_{0}\right) t}\left(\frac{e^{\left(\lambda_{0}-\mu\right) t}-e^{\left(\lambda_{0}-\mu\right) t_{s}}}{\lambda_{0}-\mu}+\frac{e^{\lambda_{0} t_{s}}}{\mu e^{\mu t}}-\frac{e^{\lambda_{0} t_{s}}}{\mu e^{\mu t_{s}}}\right) \\
& +\frac{\psi_{0} d_{1}}{\lambda_{0} \beta}\left(1-e^{\lambda_{0}\left(t_{s}-t\right)}\right)+\left\|\tilde{\phi}\left(t_{s}\right)\right\| \\
& +\mu\left\|\tilde{\phi}\left(t_{s}\right)\right\| e^{\left(\mu-\lambda_{0}\right)} \frac{e^{\left(\lambda_{0}-\mu\right) t}-e^{\left(\lambda_{0}-\mu\right) t_{s}}}{\lambda_{0}-\mu}
\end{aligned}
$$

It can be seen from (49) that if $\beta \lambda_{0}>\psi_{0} d_{0}$, then $\lambda_{0}-\mu>0$ and $\tilde{\phi}(t)$ remains bounded for $t \geq t_{s}$.

Remark 4.3: From (49) it follows $\|\tilde{\phi}(t)\| \leq \frac{\psi_{0} d_{1}}{\lambda_{0} \beta}+\left\|\tilde{\phi}\left(t_{s}\right)\right\|$ as $t \rightarrow \infty$. Since $\beta \lambda_{0}>\psi_{0} d_{0}, \psi_{0}<\frac{\beta \lambda_{0}}{d_{0}}$ and therefore $\|\tilde{\phi}(t)\| \leq \frac{d_{1}}{d_{0}}+\left\|\tilde{\phi}\left(t_{s}\right)\right\|$ as $t \rightarrow \infty$. Consequently, the residual set of $\|\tilde{\phi}(t)\|$ can be made small enough if $d_{0} \gg d_{1}$ is satisfied in (42) and the initial values of $\hat{h}(t)$ and $\hat{w}(t)$ are chosen to be close enough to their true values, which implies that $\|\tilde{\phi}(t)\|$ at time instant $t_{s}$ is small.

\section{NUMERICAL EXAMPLE}

Consider the dynamics of an aileron from JAXA's experimental MuPAL- $\alpha$ aircraft [27] given by

$$
\begin{aligned}
& \dot{x}(t)=-11.11 x(t)+w u(t-h) \\
& y(t)=9.45 x(t)
\end{aligned}
$$

which will initially be treated as the nominal model for the simulation. In this simulation, the time delay is assumed to be $h=0.3 s$. Furthermore, it is assumed that the aileron loses $50 \%$ of its effectiveness, i.e. $w=0.5$. The problem can be written in the form of (1) in Section II.

For simplicity, in the observer design the scalar modulation gain $k(t)$ from (7) is chosen to be fixed and assigned the value 19. The scalar $\lambda_{0}$ from (23), (24) and (25) is selected as 1 . In (34), the gain $\rho=100$ which governs the convergence rate of the estimation error.

In this example, the control input $u(t)$, shown in Fig. 1(a), provides sufficient excitation for the adaptive scheme. Here the control signal is chosen to be a sinusoidal signal with frequency $2 \mathrm{rad} / \mathrm{sec}$ and amplitude $0.05 \mathrm{rad}$. The disturbance signal $d(t)$ represents Gaussian white noise with a maximum amplitude of $0.05 \mathrm{rad}$. Clearly, the amplitude of disturbance is same as that of the input signal which needs to be suppressed by the proposed scheme.

Here two scenarios $(d(t)=0$ and $d(t) \neq 0)$ are considered. The plant and observer output error $e_{y}$ is shown in Fig. 1(b) which shows that the sliding occurs rapidly and the switching function is maintained close to zero despite a high frequency sinusoidal input and the disturbance $d(t) \neq 0$.

The error between the actuator fault and its estimates (i.e. $\tilde{w})$ is shown in Fig. 1(c) and Fig. 1(d) which indicates that the observer has managed to provide a good estimation of the actual loss of effectiveness despite the disturbance. This demonstrates that the approach in this paper is capable of suppressing the effect of the external disturbance. It can also be seen from Fig. 1(e) and Fig. 1(f) that despite the input delay is chosen to be 0.3 (which represents a relatively large input delay) from the beginning of the simulation, the estimation error $\tilde{h}$ converge close to zero even in the presence of the large external disturbance.

To compare the fault estimation performance of the proposed scheme and the conventional adaptive law in [8] discussed in Remark 3.1, both are plotted in Fig. 1(g). Similarly, the performance comparison for $\tilde{h}$ is shown in Fig. 1(h). It can be seen from Fig. 1(g) and Fig. 1(h), by using the conventional scheme, oscillations appear in estimates due to the limit cycle behaviour of the sliding motion. Although the convergence speed can be increased by enlarging the learning rate, the large learning rate enlarges the oscillations in the estimates as well.
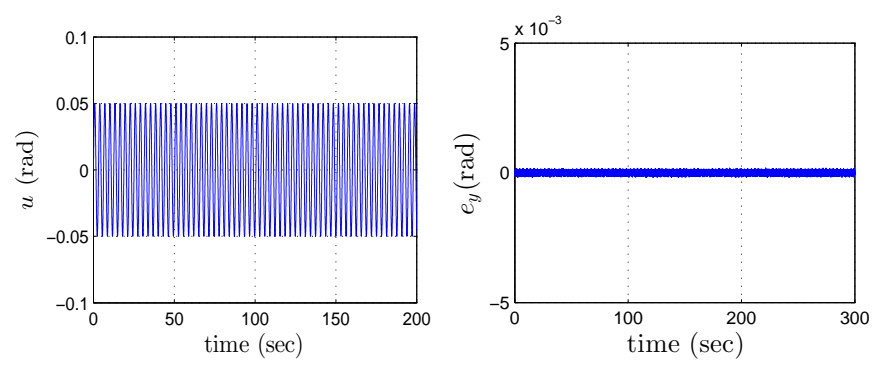

(a) control input $u(t)$

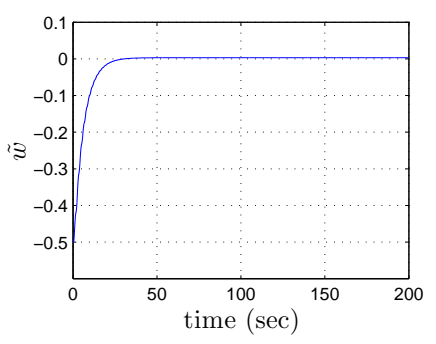

(b) estimation error $e_{y}(d(t) \neq 0)$

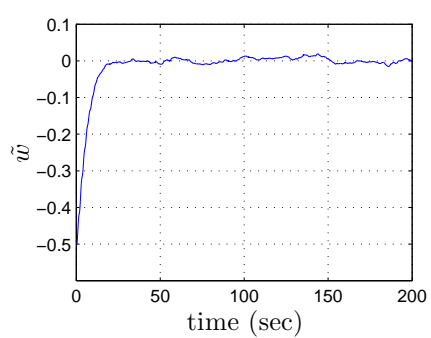

(c) estimation error $\tilde{w}(d(t)=0)$

(d) estimation error $\tilde{w}(d(t) \neq 0)$

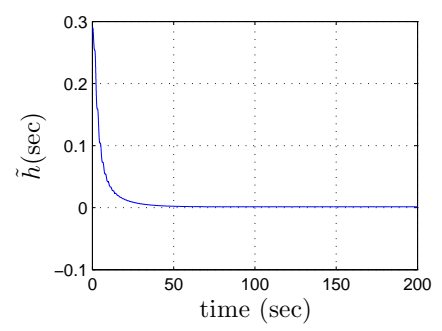

(e) estimation error $\tilde{h}(d(t)=0)$

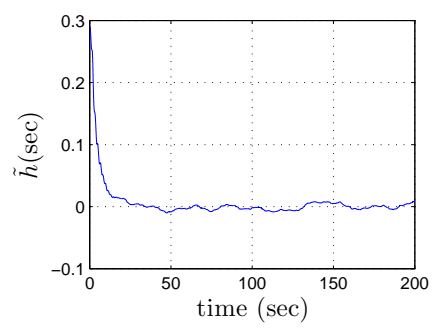

(f) estimation error $\tilde{h}(d(t) \neq 0)$
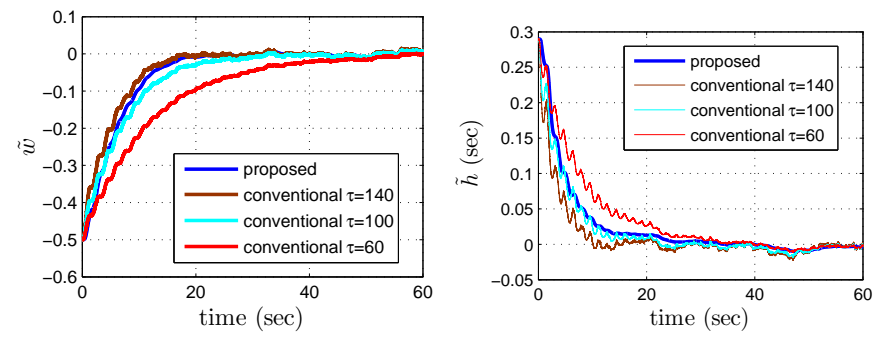

(g) comparison against the conven- (h) comparison against the conventional adaptive law on $\tilde{w}$ tional adaptive law on $\tilde{h}$

Fig. 1. Simulation results 


\section{PERFormance VAlidAtion FROM Flight teSt DATA}

To further test the new FDI scheme proposed in this paper, aircraft flight test data, collected from JAXA's experimental MuPAL- $\alpha$ aircraft from one of a series of actual flight tests conducted in January 2017 [30] will be used in an offline evaluation. During the flight tests, a fault in the aileron was introduced giving a reduction in efficiency of $50 \%$. For safety reasons, the fault was introduced at a software level. During the flight tests, air data inertial reference system (ADIRS) data and actuator performance levels were logged for monitoring and evaluation purposes [30].

As argued in [4], the aileron dynamics can be represented as a serially connected system involving a first order dynamic and a delay model. The aileron dynamic without time delay, associated with this flight condition, was modelled as in (50) used for the numerical simulation. The input time delay is uncertain and it satisfies $h \in\left[\begin{array}{ll}0.06 & 0.40\end{array}\right]$ which was identified from step and sinusoidal response data recorded from earlier flight tests [4]. During the flight test, a coordinated 'S-turn' manoeuvre, with a roll angle of $\pm 20 \mathrm{deg}$ was introduced by the pilot. The actuator commands and their surface deflections are shown in Fig. 2(b). It can be seen from Fig. 2(b) that the input time delay exists and the aileron only operates at $50 \%$ efficiency due to the presence of the fault. The observer scheme described earlier was used off-line to estimate the delay and the fault.

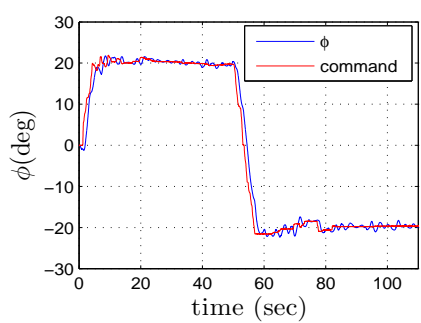

(a) roll tracking

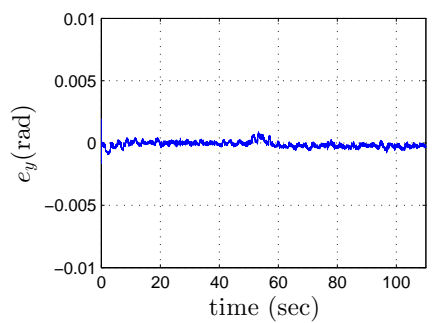

(c) sliding surface

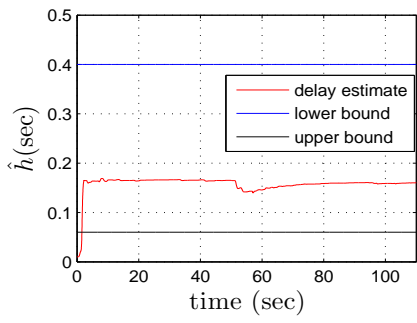

(e) delay estimation

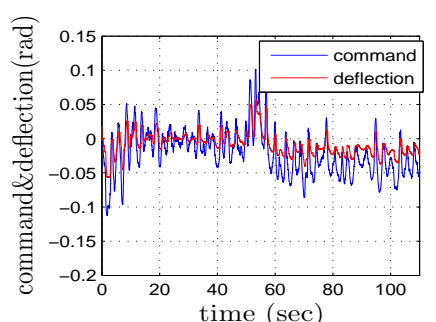

(b) command and surface deflection

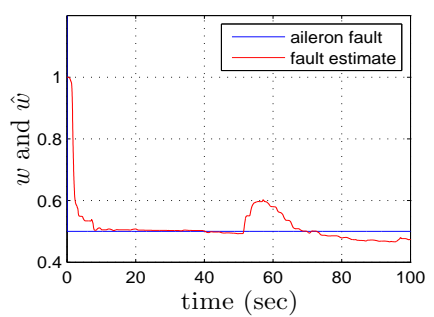

(d) fault estimation

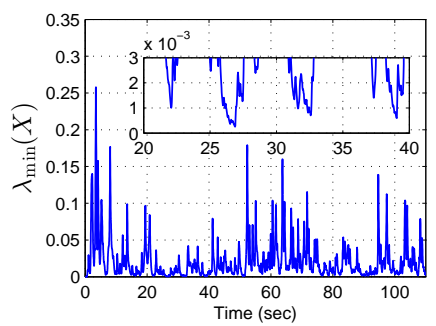

(f) $\lambda_{\min }(X(t))$
Fig. 2. flight data validation
As shown in Fig. 2(c), the sliding surface $e_{y}$ is close to zero despite the manoeuvre created by the pilot. The aileron fault implemented during the flight test and its estimation is shown in Fig. 2(d), and it can be seen from Fig. 2(d) that the fault estimate $\hat{w}$ in the early part of the experiment approaches the actual fault $w$. Notice that in Fig. 2(d) an estimation error appears between $50 \mathrm{sec}$ and $70 \mathrm{sec}$. This is in the middle of the manoeuvre when the roll command changes from $20 \mathrm{deg}$ to $-20 \mathrm{deg}$, as shown in Fig. 2(a). The input delay estimate is shown in Fig. 2(e). Clearly from Fig. 2(e), the delay estimate converges towards $0.16 \mathrm{sec}$ which is inside the known range of the aileron input delay (i.e. $h \in\left[\begin{array}{ll}0.06 & 0.40\end{array}\right]$ ). Fig. 2(f) shows that $\lambda_{\min }(X(t))>0.0002$ and therefore the Assumption 3.1 is verified and the control input in Fig. 2(b) ensures PE.

\section{CONCLUSION}

In this paper, the problem of simultaneous estimation of the input time delay and the loss of effectiveness of a single-input single-output actuator system was addressed using a sliding mode observer. This is a challenging problem and has not yet been completely resolved in the literature. By appropriately using the equivalent output error injection signal and an adaptation law, a simple sliding mode observer scheme which can guarantee convergence of the time delay and fault estimation errors is proposed. A numerical example was provided to illustrate the effectiveness of the new result together with real flight data collected from a flight test. Very accurate results demonstrating the effectiveness of the scheme were reported.

\section{REFERENCES}

[1] H. Alwi, C. Edwards and C. P. Tan, Fault Detection and Fault-Tolerant Control Using Sliding Modes. Springer, 2011.

[2] C. Edwards and S. K. Spurgeon, Sliding Mode Control: Theory and Applications. London, U.K.: Taylor \& Francis, 1998.

[3] V. Utkin, Sliding modes in control and optimization. Springer, 1992.

[4] M. Sato and A. Satoh, "Flight control experiment of multipurposeaviation-laboratory- $\alpha$ in-flight simulator," J Guid Control Dyn, vol. 34, 2011.

[5] X. Yan and C. Edwards, "Adaptive sliding-mode-observer-based fault reconstruction for nonlinear systemswith parametric uncertainties," IEEE TIE, vol. 55, pp. 4029-4036, 2008.

[6] D. Efimov, C. Edwards and A. Zolghadri, "Enhancement of adaptive observer robustness applying sliding mode techniques," Automatica, vol. 72, pp. 53-56, 2016.

[7] A. F. de Loza, F. J. Bejarano and L. Fridman, "Unmatched uncertainties compensation based on high-order slidingmode observation," Internationl Journal of Robust and Nonlinear Control, 23, 754-764, 2013.

[8] S. Sastry and M. Bodson, Adaptive Control: Stability, Convergence and Robustness. London: Prentice-Hall, 1989.

[9] D. Etter and S. Stearns, "Adaptive estimation of time delays in sampled data systems," IEEE Transactions on Acoustics, Speech, and Signal Processing, vol. 29, pp. 582-587, 1981.

[10] X. M. Ren, A. B. Rad, P. T. Chan and W. L. Lo, "Online identification of continuous-time systems with unknown time delay," IEEE TAC, vol. 50, no. 9, pp. 1418-1422, 2005.

[11] S. Diop, I. Kolmanovsky, P.E. Moraal and M. van Nieuwstadt, "Preserving stability/performance when facing an unknown time-delay," Control Engineering Practice, vol. 9, pp. 1319-1325, 2001.

[12] Y. Deng, V. Lechappe, E. Moulay and F. Plestan, "State feedback control and delay estimation for LTI system with unknown input-delay", International Journal of Control, 2020.

[13] J. Herrera and A. Ibeas, "On-line delay estimation for stable, unstable and integrating systems under step response", ISA Transactions, Vol. 51, pp. 351-361, 2012

[14] L. Belkoura, "Identifiabilty of systems described by convolution equations", Automatica, vol. 41, pp. 505-512, 2005. 
[15] J. Na, X. Ren and Y. Xia, "Adaptive parameter identification of linear SISO systems with unknown time-delay", Systems \& Control Letters, vol. 66, pp. 43-50, 2014,

[16] M. N. Mahyuddin, J. Na, G. Herrmann, X. Ren and P. Barber, "Adaptive observer-based parameter estimation with application to road gradient \& vehicle mass estimation", IEEE TIE, vol. 61, pp. 2851-2863, 2014.

[17] Y. Zhu, M. Krstic, and H. Su, "Adaptive global stabilization of uncertain multi-input linear time-delay systems by PDE full-state feedback", Automatica, vol. 96, pp. 270-279, 2018.

[18] M. Krstic and D. Bresch-Pietri, "Delay-adaptive full-state predictor feedback for systems with unknown long actuator delay", American Control Conference, pp. 4500-4505, 2009.

[19] G Zheng, A. Polyakov and A. Levant, "Delay estimation via sliding mode for nonlinear time-delay systems", Automatica, vol. 89, 2018.

[20] Y. Deng, V. Lechappe, S. Rouquet, E. Moulay and F. Plestan, "SuperTwisting Algorithm-Based Time-Varying Delay Estimation With External Signal", IEEE TIE, vol. 67, pp. 10663-10671, 2020.

[21] F. Cacace, F. Conte, A. Germani and G. Palombo, 'A new method for the simultaneous estimation of state and delay in time delay systems", IEEE CDC, pp. 5666-5670, 2015.

[22] V. Lechappe, J. De Leon, E. Moulay, F. Plestan and A. Glumineau, "Delay and state observer for SISO LTI systems", American Control
Conference, pp. 4585-4590, 2015.

[23] V. Lechappe, J. De Leon, E. Moulay, F. Plestan and A. Glumineau, "Delay and state observation for SISO nonlinear systems with input delay", Int J Robust Nonlinear Control, vol. 28, pp. 2356-2368, 2018.

[24] X. Wu, Z Sun, F. Liang and C. Yu, "Online estimation of unknown delays and parameters in uncertain time delayed dynamical complex networks via adaptive observer", Nonlinear Dynamics, vol. 73, 2013.

[25] S.V. Drakunov, W. Perruquetti, J.-P. Richard and L. Belkoura, "Delay identification in time-delay systems using variable structure observers", Annual Reviews in Control, vol. 30, pp. 143-158, 2006.

[26] A. Levant, "Robust exact differentiation via sliding mode technique," Automatica, vol. 34, pp. 379-384, 1998.

[27] M. Sato, "Robust Gain-Scheduled Flight Controller for an In-Flight Simulator," IEEE Trans. Aerosp. Electron. Syst, vol. 56, 2020.

[28] H. Khalil, Nonlinear systems (3rd Edition). Prentice Hall, 2002.

[29] L. Chen, C. Edwards and H. Alwi, "Sensor fault estimation using LPV sliding mode observers with erroneous scheduling parameters", Automatica, 101, 2019.

[30] L. Chen, H. Alwi, C. Edwards and M. Sato, "Flight evaluation of a sliding mode online control allocation scheme for fault tolerant control," Automatica, 114, 2020. 